\title{
A precancerous skin lesion that is often misdiagnosed
}

David M Ng, Roland Brand

\section{Background \\ Disseminated superficial actinic porokeratosis (DSAP) is a precancerous skin condition often seen by dermatologists that is characterised by multiple annular hyperkeratotic lesions on sun-exposed areas. In Australia, DSAP is not a rare condition, possibly owing to Australia's high levels of sun exposure, and patients with DSAP often initially present to general practitioners (GPS). A lack of awareness about DSAP may result in misdiagnosis and inappropriate management of the condition.}

\section{Objective}

The aim of this article is to provide an overview of DSAP relevant to GPs to better facilitate diagnosis, management and referral to a dermatologist.

\section{Discussion}

The underlying pathophysiological mechanism of DSAP is unknown. Although there is currently no effective standardised treatment, ongoing management is warranted, given the potential for malignant transformation. Suggested strategies include frequent full skin checks, patient education about sun protection and ablative treatment (such as cryotherapy) if indicated. Suspicious lesions require excision and histopathology.
DISSEMINATED SUPERFICIAL ACTINIC POROKERATOSIS (DSAP) is a skin disorder of keratinisation that is not uncommonly diagnosed in dermatological practice but often goes unrecognised in the community. Typically, DSAP appears in the third or fourth decade of life and is characterised by numerous erythematous or pigmented annular macules, papules or plaques with a raised hyperkeratotic border surrounding an atrophic centre. ${ }^{1,2}$ These lesions appear on sun-exposed areas such as the arms and legs and are considered precancerous.

DSAP is the most common clinical form of porokeratosis and is not a rare condition in Australia, possibly owing to Australia's high levels of sun exposure. ${ }^{3}$ In most cases, general practitioners (GPs) are the first point of contact for patients presenting with DSAP, given it is one of the most common lesions encountered during skin checks. ${ }^{4}$ Furthermore, as skin-related consultations account for $14.8 \%$ of patient encounters in general practice, ${ }^{5}$ an awareness of DSAP and its clinical features is important for appropriate diagnosis, management and referral to a dermatologist if required.

DSAP is often misdiagnosed as a rash or other skin lesion, and patients may undergo inappropriate treatment without the correct diagnosis, which is detrimental given the small risk of progression to squamous cell carcinoma (SCC), basal cell carcinoma (BCC) and rarely melanoma. ${ }^{6-8}$
Although the exact prevalence of DSAP is unknown, Australia has a higher incidence of actinic porokeratoses when compared with the rest of the world. ${ }^{1}$ Hence, an awareness of DSAP is an essential component of any Australian GP's basic knowledge of skin disorders.

\section{Aetiology and pathophysiology}

DSAP is an autosomal dominant inherited disorder with incomplete penetrance. ${ }^{2}$ The underlying pathophysiology of DSAP is unclear; however, genetics, ultraviolet (UV) radiation, trauma, infection and immunosuppression (including immunosuppression in transplant patients) are reported risk factors for porokeratosis. $^{2,9}$

In Australia, high levels of sun exposure are likely sufficient for most patients with an inherited tendency for DSAP to fully express the condition. ${ }^{1,3}$ The involvement of $U V$ radiation in the disease process is further implicated by case reports describing the development of DSAP in patients with psoriasis treated with light therapy (UVA and UVB). 1,10,11

In the literature, mutations in the MVK, SLC17A9 and SART3 genes have been reported as causative for DSAP, as have several loci identified in Chinese families..$^{1,12-16}$ The mechanism behind malignant transformation in DSAP is largely unknown, although it is thought to be due to chromosomal instability, 
p53 mutations or immunosuppression, and is more commonly seen in larger or long-standing porokeratotic lesions. ${ }^{2,8}$

\section{Clinical presentation and diagnosis}

DSAP may be difficult to detect as, given patients are likely to have a history of significant sun exposure, there are often co-existing findings of sun damage such as solar keratoses and pigmentary changes. In these patients, porokeratotic lesions may go unnoticed or are most often misdiagnosed as multiple solar keratoses. The differential diagnosis also includes seborrhoeic keratoses, psoriasis, discoid eczema, annular lichen planus or other rarer dermatological conditions. ${ }^{9}$

The porokeratotic lesions in DSAP begin as pink-to-brown macules and papules with a raised border on sun-exposed sites - such as the legs, forearms, shoulders, back and rarely on the face - with a bilateral and symmetrical distribution. ${ }^{2,9}$ Over time, these lesions may spread (and coalesce) in a centrifugal manner and appear as
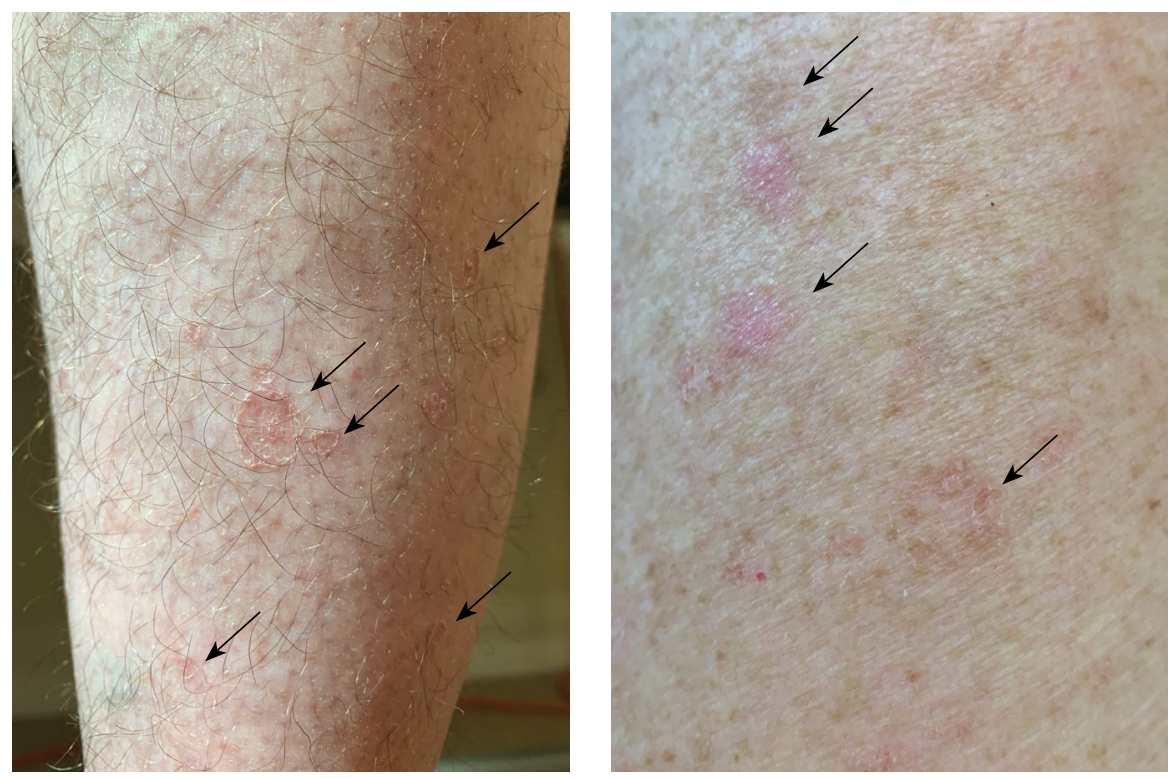

Figure 1. Disseminated superficial actinic porokeratosis lesions on the right calf and left upper arm of a male aged 52 years; arrows indicate the lesions
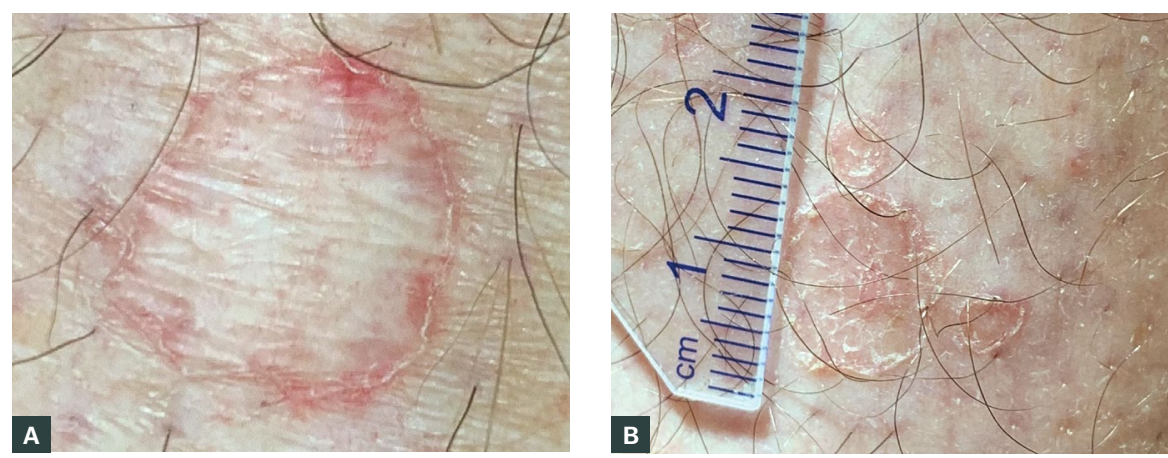

Figure 2. Dermoscopic features of porokeratotic lesions

A. Lesion displaying a white hyperkeratotic border surrounding an atrophic centre with some macular telangiectasia; B. Multiple porokeratotic lesions of various sizes with peripheral keratotic borders and a central erythematous atrophic scale

well-circumscribed annular macules or plaques consisting of an atrophic centre with a hyperkeratotic border (Figure 1). DSAP may be asymptomatic or pruritic, and lesions tend to worsen and multiply when exposed to sunlight or UV light; conversely, these lesions may fade in winter. ${ }^{1}$

DSAP is often diagnosed clinically on gross examination on the basis of the characteristic appearance of the lesion and features of the patient's history, such as sun exposure, family history or immunosuppression. Dermoscopy may prove useful in supporting the diagnosis (Figure 2), and features of DSAP on dermoscopic examination include: ${ }^{17}$

- a white peripheral border around the lesion

- a homogenous central white scar-like area

- brownish globules or dots

- vascular structures - pinpoint vessels or irregular linear vessels crossing the lesion.

Definitive diagnosis may be achieved by biopsy and histological examination (Figure 3). The characteristic hallmark of porokeratotic lesions is the cornoid lamella, which is described as a well-circumscribed vertical stack of parakeratotic keratinocytes extending through the stratum corneum. ${ }^{9}$

\section{Management}

Ongoing management of DSAP includes patient education about sun protection and regular skin checks. As previously mentioned, given the probable history of significant sun exposure, patients are likely at increased risk of skin cancer both dependent and independent of DSAP. Lesions suspicious for malignancy should be excised and sent for histological examination. Given the autosomal dominant transmission of DSAP, family screening may be useful to identify others with DSAP and implement appropriate preventive and management strategies.

Treatment of DSAP may be required given the potential progression to malignancy or if the patient desires medical treatment. To date, a number of treatments have been proposed and used with variable success, although 
the majority of the evidence supporting these comes from case series or reports. These treatments have included the use of topical diclofenac, photodynamic therapy, vitamin D3 analogs (calcipotriol), 5-fluorouracil, retinoids (adapalene), imiquimod, cryotherapy, excision, curettage, dermabrasion and laser therapies including Grenz ray therapy. ${ }^{18-20}$ In their clinical experience, these authors have found success in using ablative measures for DSAP, with the most practical being cryotherapy, followed by laser. However, there is currently no evidencebased standardised treatment protocol for DSAP, and further research to evaluate treatments for DSAP are required.

Overall, given the increased risk of malignancy and uncertain future of DSAP treatments, referral to a dermatologist is recommended on suspicion or diagnosis of DSAP in order to facilitate ongoing management and treatment.

\section{Key points}

- DSAP is the most common porokeratosis and is more common in countries with high sun exposure, such as Australia.
- The porokeratotic lesions in DSAP may be asymptomatic or pruritic and predominantly appear on sun-exposed areas, such as the arms and legs, as multiple annular lesions with raised hyperkeratotic borders. These lesions are considered precancerous and have a small risk of malignant transformation.

- Diagnosis is usually made clinically, although histology can confirm the diagnosis.

- The management for DSAP includes patient education on sun protection, regular full skin checks and consideration of specific treatment for each lesion.

- DSAP is an autosomal dominant condition and family screening is useful to detect further cases.

- There is no standardised evidencebased treatment for DSAP, although various treatments have been used by dermatologists with variable success.

\section{Authors}

David M Ng BSc, final-year medical student (MD), The University of Western Australia

Roland Brand MBBS, FACD, FRACP, Consultant Dermatologist and Physician, Head of

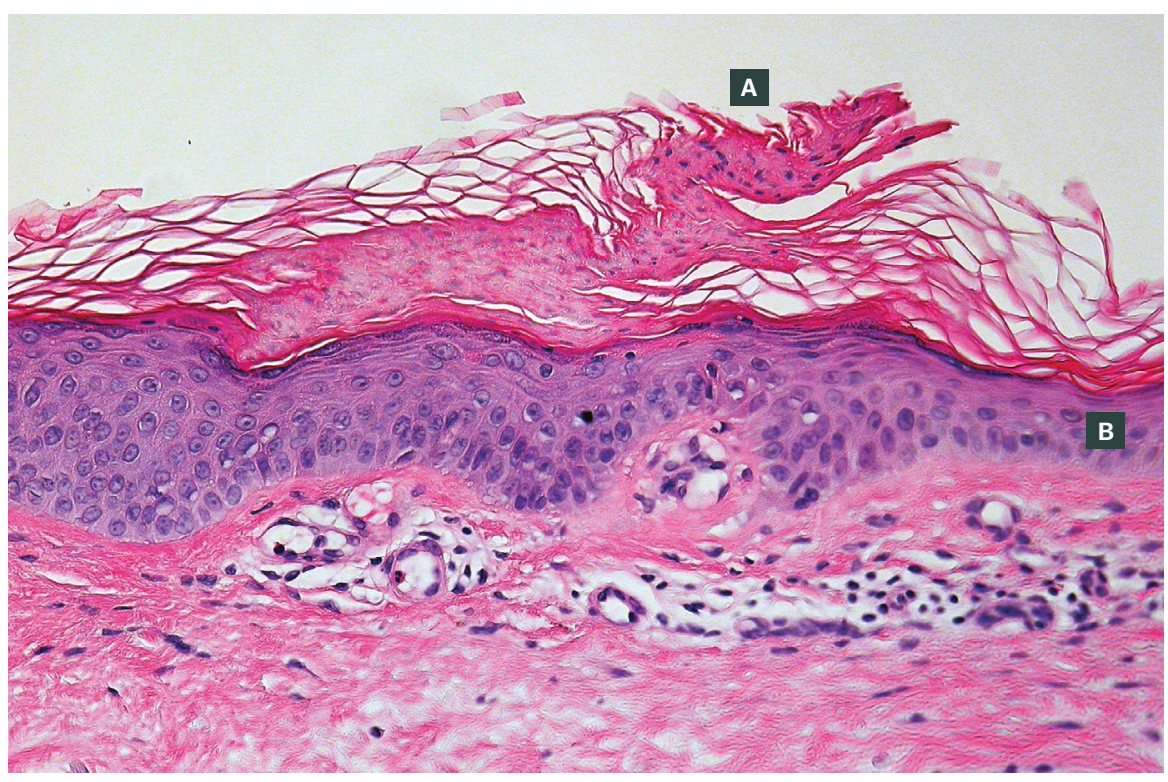

Figure 3. Histopathology of a porokeratotic lesion in disseminated superficial actinic porokeratosis A. A central porokeratotic cornoid lamella is shown overlying dyskeratotic keratinocytes; B. Surrounding areas of epidermal atrophy
Dermatology, Perth Children's Hospital, WA. drbrand@optusnet.com.au

Competing interests: None.

Funding: None.

Provenance and peer review: Not commissioned, externally peer reviewed.

\section{Acknowledgements}

The authors would like to acknowledge Professor Lawrence Yu (Dermatopathologist) for his contribution to Figure 3.

\section{References}

1. Kanitakis J. Porokeratoses: An update of clinical, aetiopathogenic and therapeutic features. Eur J Dermatol 2014;24(5):533-44. doi: 10.1684/ ejd.2014.2402.

2. Le C, Bedocs PM. Disseminated superficial actinic porokeratosis. Treasure Island, FL: StatPearls Publishing, 2018.

3. Shumack SP, Commens CA. Disseminated superficial actinic porokeratosis: A clinical study. J Am Acad Dermatol 1989;20(6):1015-22. doi: 10.1016/s0190-9622(89)70126-2.

4. Sinclair R. Skin checks. Aust Fam Physician 2012;41(7):464-69.

5. Britt H, Miller GC, Charles J, et al. General practice activity in Australia 2000-01 to 2009-10: 10 year data tables. General practice series no. 28. Cat. no. GEP 28. Canberra: AlHW, 2010.

6. Lee HR, Han TY, Son SJ, Lee JH. Squamous cell carcinoma developing within lesions of disseminated superficial actinic porokeratosis. Ann Dermatol 2011;23(4):536-38. doi: 10.5021/ ad.2011.23.4.536.

7. Riyaz N. Porokeratosis and malignancy: Incidental or causal association? Indian Dermatol Online J 2015;6(6):452-53.

8. Al-Haseni A, Chitgopeker P, Ho JD, Goldberg LJ, Sahni D. Amelanotic melanoma arising within a lesion of disseminated superficial actinic porokeratosis: An unusual presentation leading to a novel therapeutic approach. Dermatol Ther 2018;31(1):e12552. doi: 10.1111/dth.12552.

9. Sertznig P, von Felbert V, Megahed M. Porokeratosis: Present concepts. J Eur Acad Dermatol Venereol 2012;26(4):404-12. doi: 10.1111/j.1468-3083.2011.04275.x.

10. Kawara S, Oiso N, Kawada A. Disseminated superficial actinic porokeratosis in a patient undergoing treatment with long-term narrowband ultraviolet $B$ for psoriasis. J Dermatol 2011;38(6):585-87. doi: 10.1111/j.13468138.2010.01099.x.

11. Allen $A L$, Glaser DA. Disseminated superficial actinic porokeratosis associated with topical PUVA. J Am Acad Dermatol 2000;43(4):720-22. doi: 10.1067/mjd.2000.107941.

12. Zhang ZH, Niu ZM, Yuan WT, et al. A mutation in SART3 gene in a Chinese pedigree with disseminated superficial actinic porokeratosis. $\mathrm{Br} J$ Dermatol 2005;152(4):658-63. doi: 10.1111/j.1365-2133.2005.06443.x.

13. Dereure O. Mutation in the SLC17A9 gene in familial superficial actinic disseminated porokeratosis. Ann Dermatol Venereol 2015;142(2):155-56. doi: 10.1016/j. annder.2014.12.004.

14. Zhu T, Tian D, Zhang L, et al. Novel mutations in mevalonate kinase cause disseminated superficial actinic porokeratosis. Br J Dermatol 2018 181(2):304-13. doi: 10.1111/bjd.17596. 
15. Xia JH, Yang YF, Deng $H$, et al. Identification of a locus for disseminated superficial actinic porokeratosis at chromosome 12q23.2-24.1.

$\mathrm{J}$ Invest Dermatol 2000;114(6):1071-74. doi: 10.1046/j.1523-1747.2000.00978.x.

16. Luan J, Niu Z, Zhang J, et al. A novel locus for disseminated superficial actinic porokeratosis maps to chromosome 16q24.1-24.3. Hum Genet 2011;129(3):329-34. doi: 10.1007/s00439-0100929-x.

17. Nicola A, Magliano J. Dermoscopy of disseminated superficial actinic porokeratosis. Actas

Dermosifiliogr 2017;108(5):e33-e37. doi: 10.1016/j. ad.2015.09.025.

18. Skupsky H, Skupsky J, Goldenberg G. Disseminated superficial actinic porokeratosis: A treatment review. J Dermatolog Treat 2012;23(1):52-56. doi: 10.3109/09546634.2010.495381.

19. Park BJ, Oh EH, Kim JE, Ko JY, Ro YS. Treatment of disseminated superficial actinic porokeratosis with oral alitretinoin. J Eur Acad Dermatol Venereol 2017;31(11):e505-e507. doi: 10.1111/jdv.14354.

20. Weidner T, Illing T, Miguel D, Elsner P. Treatment of porokeratosis: A systematic review. Am J Clin Dermatol 2017;18(4):435-49. doi: 10.1007/s40257-

017-0271-3. 